\title{
Georges VIGARELLO, Histoire de la fatigue du Moyen Âge à nos jours
}

Jean-Paul Callède

\section{OpenEdition}

1 Journals

Édition électronique

URL : https://journals.openedition.org/ress/7240

DOI : $10.4000 /$ ress. 7240

ISBN : $1663-4446$

ISSN : $1663-4446$

Éditeur

Librairie Droz

Édition imprimée

Date de publication : 21 mai 2021

Pagination : 257-261

ISSN : 0048-8046

Référence électronique

Jean-Paul Callède, "Georges VIGARELLO, Histoire de la fatigue du Moyen Âge à nos jours ", Revue européenne des sciences sociales [En ligne], 59-1 | 2021, mis en ligne le 21 mai 2021, consulté le 25 mai 2021. URL : http://journals.openedition.org/ress/7240 ; DOI : https://doi.org/10.4000/ress.7240

Ce document a été généré automatiquement le 25 mai 2021.

(c) Librairie Droz 


\title{
Georges VIGARELLO, Histoire de la fatigue du Moyen Âge à nos jours
}

\author{
Jean-Paul Callède
}

\section{RÉFÉRENCE}

Georges VIGARELLO, 2020, Histoire de la fatigue du Moyen Âge à nos jours, Paris, Éditions du Seuil, 476 p.

1 L'ouvrage que Georges Vigarello vient de publier aux Éditions du Seuil est de première importance et, pourrait-on ajouter, de circonstance. L'auteur examine l'intérêt progressif porté à la fatigue depuis le Moyen Âge jusqu'à nos jours. Au cours des siècles, en Occident, la fatigue s'impose peu à peu comme un thème de préoccupations sociales, culturelles mais aussi scientifiques, au regard de la santé, de l'économie, jusqu'à justifier des intérêts d'ordre juridique et des arbitrages politiques. Il s'agit de rendre compte de l'importance grandissante prise par la question de la fatigue, rapportée à la vie collective organisée autour des groupes sociaux, de la transformation des techniques et des modèles du travail, sans oublier les guerres qui ont jalonné, à leur façon, la longue durée.

2 Aujourd'hui, avec son sillage d'attributs ou de qualificatifs, la fatigue est très présente dans le vocabulaire et dans les faits. «Elle s'impose dans l'espace public, au travail, dans l'espace privé, dans les relations avec les proches comme dans les relations de soi à soi. » (Introduction, p. 9). Pour autant, pareille évidence ne saurait satisfaire l'historien. En effet, aborder la réalité de la fatigue depuis le Moyen Âge relève même du défi, plus exactement d'une série ordonnée de défis si l'on se réfère à l'intitulé des cinq parties qui composent le livre. Les sources visitées sont multiples, caractéristiques des époques traversées. Le corpus des notes bibliographiques, classé par chapitres, occupe les pages 375-456 et l'index des noms de personnes, les pages 457-470. Ceci reflète le sérieux de la recherche documentaire. 
3 La première partie envisage «l'univers médiéval et le défi des repères ». L'auteur constate qu'il est possible de relever des expressions verbales, des situations qui supposent un intérêt ponctuel sur la fatigue et ses manifestations. Le chapitre qui ouvre l'étude: "Image claire, repères incertains »(chap. 1), précise les informations sur lesquelles l'historien est censé étayer son analyse tandis qu'une image centrale commande la vision ancienne de la fatigue : celle de la perte, d'un affaiblissement de la personne dans ce que ces aspects peuvent avoir de plus visuel. Les situations caractéristiques en sont: «La fatigue célébrée du combattant» (chap.2), «La souffrance "obligée" du voyageur» (chap.3) confronté aux « risques des itinéraires tourmentés» (p. 29). Ces formes de voyages diffèrent de celles relevant de l'univers religieux, tels les pélerinages et autres «contritions charnelles » ( La fatigue "rédemptrice" ", chap. 4). L'auteur considère que le travail quotidien est une sorte de point aveugle tant la fatigue n'y est jamais ou peu nommée (chap. 5). Il en propose une explication: trivialité du rural, qui prolonge initialement le statut de serf des $\mathrm{X}^{\mathrm{e}}$ et $\mathrm{XI}^{\mathrm{e}}$ siècles mais aussi intérêt porté aux outils et autres « engins » dont l'homme se sert, plutôt qu'à la pénibilité liée à leur utilisation. Pourtant, les villes, qui favorisent l'essor de différents métiers, sont propices ici ou là à la prise de conscience de la durée du travail, y compris chez des travailleurs de peine, avec les griefs que suscitent certains litiges. Un dernier chapitre: "Entre force occulte et vertus du rafraîchissement » (chap. 6) regroupe tout un ensemble de « recettes » : boissons, épices, etc., mais aussi «talismans » associés à des imaginaires ou des puissances occultes.

«L'univers moderne et le défi des catégories » fait l'objet de la deuxième partie. Déjà se dessine, dans les textes exploités, une intention de mieux caractériser des états jusquelà négligés (« Inventer des degrés », chap. 7). L'auteur entre dans le détail avec les deux chapitres suivants: "Inventer des catégories » (chap. 8) et "L'amorce du chiffre » (chap.9), avant d'examiner la manière dont des témoins instruits relatent leurs impressions («Une diversification des effets", chap.10): " engoudissements", incommodations physiques, épuisement sexuel. Les efforts ouvriers et leurs conséquences sont à peine relevés. En matière de prévention et de remèdes («Une diversification des "recours"», chap.11), un idéal demeure, centré sur le fonctionnement du corps : " préserver les liquides, restaurer les humeurs» (p. 97), qui intègre le "tabac » et le "café ». La panoplie des fatigues qui se précise aux $\mathrm{XVI}^{\mathrm{e}}$ et XVII ${ }^{\mathrm{e}}$ siècles s'impose avec les «lassitudes » que l'élite classique lettrée a inventées. Cependant, l'examen de la correspondance des intendants et de certains notables tend à montrer la misère des gens de peu, dans les villes et les campagnes (chap.12), aggravée par le poids de l'impôt (p. 108).

5 La partie qui suit envisage «les Lumières et le défi du sensible». Au cours du XVIII ${ }^{\mathrm{e}}$ siècle, l'approfondissement d'une explication de la fatigue conduit à explorer de nouvelles perspectives. Deux constats s'imposent. "L'enjeu du sensible » (chap. 13) suppose une exploration introspective que la «science» permet de nommer, à l'exemple du Dictionnaire portatif de santé (1770). En outre, "le recul du divin » livre davantage à lui-même "l'homme "sensible" des Lumières" (p. 111). Un nouvel univers physique se déploie: "Le nerf, de l'agitation au "tourbillon"» (chap.14). Ces « questions neuves » ("Dire les forces », chap. 15), sont formulées par le monde savant sur fond d'une temporalité nouvelle: celle du progrès, tels les travaux de Bernoulli, Coulomb ou Lavoisier. Cette curiosité savante et intellectuelle s'accompagne d'une attention qui se focalise sur l'industrie, au point de transformer la vision de 
l'observateur («La fatigue "subie", amorce de compassion » (chap. 16). Dans un écrit de 1783, Diderot note : « Il y a beaucoup d'états dans la société qui excèdent la fatigue, qui épuisent promptement les forces et qui abrègent la vie » (p. 144). Les deux chapitres suivants (chap. 17 : «La fatigue "recherchée", amorce de défi » et chap. 18: «L'amorce de l'entrainement, la révision du temps ») ouvrent des perspectives plus réjouissantes. En effet, les voyages s'agrémentent de la rapidité des déplacements ou de l'appel du lointain relaté dans les publications de James Cook, de Bougainville, etc. Par ailleurs, "Le défi des sommets" et l'appel de la montagne renouvellent l'horizon des épuisements. La soif de connaissances en divers domaines s'accompagne d'un recul des limites, inaugurant de nouvelles expériences de la fatigue, parfois au péril de la vie (chap. 18).

6 La quatrième partie s'intitule : "Le XIX ${ }^{e}$ siècle et le défi du chiffre ». Le siècle du "positivisme » transforme la fatigue en un objet d'étude cultivant l'administration de la preuve. Elle s'ouvre sur un chapitre intitulé : «Le citoyen et la tenacité » (chap. 19), largement nourri de portraits empruntés à la littérature. Le chapitre qui suit : «Un univers chiffré: de la mécanique à l'énergie» (chap. 20) confirme l'essor de la mécanique industrielle et le regard des mécaniciens se tourne vers la physiologie. D'ailleurs les travaux sur l'alimentation eux-mêmes s'orientent de la simple hygiène de vie vers l'étude de la dépense énergétique, inspirant le régime alimentaire des lycées ou de l'armée. Pour autant, le culte du rendement et du chiffre conduisent à des excès qui sont analysés dans les trois chapitres suivants. Dans « Un univers menacé : la misère du labeur » (chap. 21), l'auteur commence par explorer la triste condition sociale et physique des gens de peine, ouvriers ou autres. «Une pensée socialiste prend forme, de Bakounine à Engels, de Proudhon à Blanqui, censée éclairer l'extrême indigence des prolétaires. Karl Marx en offre la lecture la plus suggestive, comme la plus exhaustive » (p. 206). Dans le prolongement de ces études critiques, il examine ensuite "l'univers du rendement » (chap. 22). Cette logique du rendement tend à ignorer l'effort de contrôle et d'objectivation accompli pour mieux comprendre le travail et ses effets. On peut considérer qu'« une science de la fatigue» se précise avec Angelo Mosso, dont l'ergographe permet de chiffrer et de retranscrire " pour la première fois, la fatigue selon un graphique et ses sinuosités» (p. 219). Bientôt, un autre mode d'objectivation va susciter un intérêt certain avec les "principes d'organisation du travail» de l'ingénieur Taylor, de Philadelphie, complétés par ceux de Ford dans ses usines de Detroit dès les années 1910. Quoi de plus logique alors que l'auteur consacre les deux chapitres suivants à l'« univers de la fatigue "mentale" » (chap. 23) et aux " résistances et "grandissements" » (chap. 24). On renverra le lecteur aux fines analyses que l'auteur consacre au «surmenage scolaire» (p. 244-246) ou à «la neurasthénie» (p. 247-250), maux caractéristiques de l'époque. La place des activités sportives prend le relai des gymnastiques proposées au cours de la première moitié du XIX siècle.

7 La cinquième et dernière partie a pour titre: "Les $\mathrm{XX}^{\mathrm{e}}-\mathrm{XXI}^{\mathrm{e}}$ siècles et le défi du psychologique». Elle est composée de quatre chapitres dont le premier «Révéler le psychique " (chap. 25) indique un bornage majeur. La Première Guerre mondiale a engendré "des fatigues extrêmes, des formes d'épuisement jusque-là inconnues » (p. 277). Dans les années qui suivent, le travail fait l'objet de nouvelles dispositions. La "médecine du travail » voit le jour (p. 282). Il s'agit également de revisiter les lieux et les temps du travail, à l'exemple du protocole expérimental établi par Elton Mayo à la Western Electric de Cicero, près de Chicago, entre 1922 et 1932. La crise mondiale sert de révélateur. "Abattement », « dépression », « cafard » sont autant de façons de désigner 
une fatigue qui ne dit pas son nom. Dans le domaine de la biologie, de nouvelles références se précisent, qui font l'objet du chapitre suivant : « Des hormones au stress " (chap. 26). Avec la découverte des amphétamines, des «rêves d'infatigabilité " se précisent (p.301). En vente libre sous brevet américain dès les années 1930, la « benzédrine » se diffuse. Les sportifs utilisent le produit, les intellectuels le banalisent, les militaires s'y intéressent avant que son usage ne se répande sur les champs de bataille de la Seconde Guerre mondiale (p. 303). Au milieu des années 1930, un mot emprunté aux travaux sur la résistance des matériaux, "Repeated Stress ", désigne les perturbations de la personne associées à une intensification des sécrétions hormonales sous l'effet d'une « agression » ou de " chocs » répétés. Ces avancées des connaissances ont prise sur certains régimes politiques totalitaires ("De l'"homme nouveau" au tragique », chap. 27). L'auteur s'intéresse entre autres à la question des loisirs (p.313-315), qui revêt une signification différente selon les totalitarismes et les démocraties. Avec les deux derniers chapitres, l'auteur aborde la seconde moitié du $\mathrm{XX}^{\mathrm{e}}$ siècle. Espérances sociales portées par la prospérité économique, avec "Les promesses du bien-être?» (chap. 28) et atteintes à l'autonomie des individus avec le dernier chapitre : «Du burn out à l'identité » (chap. 29). Le souffle d'espérance né de la fin du conflit mondial, conforté par des mesures législatives innovantes, se prolonge dans des recherches qui croisent différents modèles censés prévenir chez l'individu le désordre et la fatigue. Pour autant, en dénonçant le «travail en miettes », avec Georges Friedmann, d'autres approches considèrent l'ambivalence qui se joue entre prospérité économique, asservissement au travail et bien-être personnel. Les fatigues, à l'exemple de la "nouveauté des "troubles musculo-squelettiques" » (p. 336), conduisent à revoir les nomenclatures des "pénibilités au travail» qu'un ministère du Travail tend à simplifier, au mépris des témoignages récurrents et des publications de référence. En effet, du livre "inaugural»Stress et satisfaction au travail: étude critique (Bureau international du travail, 1983) aux alertes de l'Académie de médecine (« Rapport sur le burn out », 2016) et au « très important Dictionnaire de la fatigue » (Droz, 2016) (p. 451), les connaissances scientifiques actualisées ne manquent pas. La "fatigue quotidienne " s'impose, «traversant les âges et les conditions » (p. 354). Dans l'intimité, l'autonomie individuelle engendre anxiété, fatigue et solitude. Le "management», la "psychologie " et autres "façons de se soigner ", voire la communication via les " réseaux sociaux ", ne sont que des réponses abrégées, incomplètes.

La «Postface » intitulée: "Surprises et menaces "virales" (p. 361-372) replace le lecteur face à sa propre expérience de la "pandémie de Covid-19, surgie à la fin de l'année 2019 » (p. 363). Elle engendre des fatigues nouvelles que l'auteur analyse à travers les échos renvoyés par les médias au cours du premier semestre 2020.

Georges Vigarello a gagné son pari ou plutôt une succession de paris attachés aux périodes qui structurent l'agencement du livre. La grille de lecture et d'analyse qu'il a patiemment édifiée pour son Histoire de la fatigue est des plus convaincantes, avec son affirmation progressive au fur et à mesure que la société se complexifie et que l'individu semble y gagner en autonomie. "Tout révèle une histoire, plus complexe qu'il n'y paraît et encore peu étudiée, riche de métamorphoses qui en signalent d'autres, mobilisant l'investissement des acteurs, des cultures, des sociétés " (Introduction, p. 10). L'ambition initiale porte ses fruits et la lecture de cette Histoire de la fatigue est une clé pertinente qui permet de questionner les sociétés occidentales 
dans leur ensemble. Le livre sera d'une grande utilité pour éclairer d'autres approches thématiques dans le champ des sciences de l'homme et de la société.

\section{AUTEURS}

\section{JEAN-PAUL CALLĖDE}

Bordeaux, Maison des sciences de l'homme d'Aquitaine 